\title{
A Prevalência de Pré-Obesidade/Obesidade nas Crianças do Ensino Pré-Escolar e Escolar na Autarquia de Vila Nova de Gaia, Portugal
}

\section{Prevalence of Pre-Obesity/Obesity in Pre and Basic School Children at Vila Nova de Gaia, Portugal}

\author{
Bárbara CAMARINHA $\rrbracket^{1}$, Pedro GRAÇA ${ }^{2,3}$, Paulo Jorge NOGUEIRA ${ }^{4,5}$
}

Acta Med Port 2016 Jan;29(1):31-40

\section{RESUMO}

Introdução: Mais de $30 \%$ das crianças portuguesas apresentam excesso de peso. Sendo um grave problema de saúde pública com múltiplas causas, só uma ação intersectorial e concertada poderá contribuir para a sua resolução. As autarquias possuem um conjunto de competências que as tornam ideais para uma intervenção eficaz na luta contra este problema. Para essa ação ser efetiva é necessária uma avaliação local da dimensão do problema e sua distribuição espacial. O objetivo deste estudo foi caracterizar, do ponto de vista da prevalência de excesso de peso, a população do pré-escolar e primeiro ciclo do ensino básico sob responsabilidade da autarquia de Vila Nova de Gaia, o terceiro município mais populoso do país.

Material e Métodos: Avaliaram-se 8974 crianças do pré-escolar e primeiro ciclo do ensino básico do município, no ano letivo $2013 / 14$. A avaliação do estado nutricional da amostra foi feita com recurso às medidas antropométricas peso e altura. Na classificação do estado nutricional considerou-se os percentis ou z-scores do índice de massa corporal para a idade de acordo com os critérios definidos pela Organização Mundial de Saúde, Centers for Disease Control and Prevention e pela International Obesity Task Force.

Resultados: A prevalência de excesso de peso pelo critério da Organização Mundial da Saúde foi de 37,4\%. Identificaram-se disparidades na distribuição do excesso de peso nas escolas do concelho.

Discussão: Os resultados encontrados foram ligeiramente superiores aos de outros estudos nacionais e internacionais, podendo a abrangência amostral justificar as diferenças encontradas.

Conclusão: A prevalência encontrada é elevada e de distribuição aparentemente distinta a nível espacial, o que poderá contribuir para a identificação dos principais determinantes do problema a nível local.

Palavras-chave: Composição Corporal; Criança; Criança Pré-Escolar; Excesso de Peso; Índice de Massa Corporal; Obesidade Pediátrica; Portugal; Prevalência.

\section{ABSTRACT}

Introduction: Over $30 \%$ of Portuguese children present overweight. Being a serious public health problem with multiple causes, only a cross-sectorial and concerted action could contribute to their resolution. Municipalities have a set of skills that make them ideal for effective intervention in the fight against this problem. For this action to be effective there must be a local assessment of the extent of the problem and their spatial distribution. The aim of this study was to characterize, from the point of view of the prevalence of overweight, the population of pre-school and first cycle basic education under the responsibility of the municipality of Vila Nova de Gaia, the third most populous municipality in the country.

Material and Methods: Were evaluated 8974 pre and basic-school children, in school year 2013/14. The assessment of the nutritional status of the sample was made using anthropometric measures height and weight. In the classification of nutritional status was considered z-scores or percentiles of body mass index for age according to the World Health Organization, Centers for Disease Control and Prevention and International Obesity Task Force.

Results: The prevalence of overweight by the World Health Organization requirements was $37.4 \%$. There were some disparities in the distribution of overweight in schools of the municipality.

Discussion: The results were slightly higher than those of other national and international studies, being the sampling size the possible justification for the differences founded.

Conclusion: The prevalence found is high and with irregular geographical distribution, which could help identifying the main causes of the problem at the local level.

Keywords: Body Composition; Body Mass Index; Child; Child, Preschool; Overweight; Pediatric Obesity; Portugal; Prevalence.

\section{INTRODUÇÃO}

A obesidade infantil é um problema global. Em 2013, 42 milhões de crianças com menos de cinco anos de idade apresentavam excesso de peso, em todo o mundo. ${ }^{1} \mathrm{Na}$ Europa, este valor ronda os 14 milhões de crianças, às quais se juntam, anualmente, mais $400000^{2,3}$
Em Portugal, mais de $30 \%$ da população infantil apresenta excesso de peso. ${ }^{3}$ Estes valores triplicaram em muitos países europeus desde 1980, para a população em geral, e nas crianças chegam a ser 10 vezes superiores aos registados em 1970, ${ }^{3}$ colocando a obesidade como um

1. Departamento de Educação. Câmara Municipal de Vila Nova de Gaia. Vila Nova de Gaia. Portugal.

2. Faculdade de Ciências da Nutrição e Alimentação. Universidade do Porto. Porto. Portugal.

3. Programa nacional para a Promoção da Alimentação Saudável. Direção-Geral da Saúde. Lisboa. Portugal.

4. Faculdade de Medicina. Universidade de Lisboa. Lisboa. Portugal.

5. Serviço de Informação e Análise. Direção-Geral da Saúde. Lisboa. Portugal.

$\bowtie$ Autor correspondente: Bárbara Camarinha. barbaracamarinha@gmail.com

Recebido: 08 de junho de 2015 - Aceite: 17 de agosto de 2015 | Copyright @ Ordem dos Médicos 2016 
problema de saúde pública de proporções epidémicas a nível mundial. ${ }^{4-6}$ Segundo Marie $\mathrm{Ng}$ et al, ${ }^{7}$ contavam-se em 2013 cerca de 2,1 biliões de indivíduos com excesso de peso em todo o mundo, enquanto em 1980 este valor era de 857 milhões. $^{7}$

A Organização Mundial da Saúde (OMS), que em 2002 considerou a obesidade como sendo um dos dez principais fatores de risco de mortalidade, ${ }^{8}$ refere-a em 2004 como a epidemia do século XXI. ${ }^{9}$ Atualmente, o número de pessoas com excesso de peso é 2,5 vezes superior ao número de pessoas subnutridas. ${ }^{10}$ Deve contudo salientar-se que nos países de alto rendimento se tem vindo a assistir a uma desaceleração deste crescimento,, 711 o que não se verifica nos países de baixo e médio rendimento, que se debatem com os dois extremos do dilema da malnutrição: a subnutrição e o excesso de peso. ${ }^{2}$

A obesidade tem etiologia multifatorial, com determinantes hereditários, genéticos, ambientais, metabólicos, comportamentais, culturais e socioeconómicos os quais interagem e se potenciam. Caracteriza-se por um excesso de peso corporal, com elevada acumulação de gordura, devido à manutenção de um balanço energético positivo ao longo do tempo. ${ }^{12,13}$ Está descrito na literatura que alguns dos potenciadores do desenvolvimento da obesidade infantil incluem ter pais obesos, ter baixo estatuto socioeconómi$c 0$, apresentar elevado peso à nascença, ter um estilo de vida sedentário e padrões alimentares inadequados. ${ }^{14,15}$

Se por um lado, o organismo apresenta excelentes defesas fisiológicas contra a depleção das suas reservas energéticas, por outro, apresenta frágeis meios de combate à acumulação excessiva de reservas energéticas, em situações de abundância de alimento. ${ }^{16}$

Alguns estudos sugerem que o excesso de peso durante a infância é um fator de risco para o desenvolvimento de obesidade na idade adulta, calculando-se que cerca de $60 \%$ das crianças que se apresentem nestas condições antes da puberdade, manterão, enquanto jovens adultos, o excesso de peso, com efeitos adversos a curto e a longo prazo, que afetam a morbilidade e a mortalidade do adulto. ${ }^{9,12,13,17,18}$ As consequências da obesidade durante a infância e adolescência, incluem não só problemas a nível de saúde física, como hipertensão arterial, hipercolesterolemia, síndrome metabólica, diabetes tipo 2, problemas osteoarticulares, apneia do sono, asma e cirrose hepática, mas também consequências a nível psicológico, social e comportamental, como baixa autoestima, isolamento social, insucesso escolar, descriminação, depressão e diminuição da qualidade de vida em geral. ${ }^{13}$ Consequentemente, a avaliação e intervenção precoces, ainda durante a infância, tornam-se fundamentais para prevenir a disseminação desta epidemia e comorbilidades associadas.

Não obstante a intervenção/tratamento individual, a resolução deste problema deve assentar, essencialmente, numa estratégia de prevenção. ${ }^{19}$ Prevenção essa, que será tanto mais eficaz quanto mais precoce for o seu início, em termos de idade. ${ }^{20} \mathrm{~A}$ infância é considerada como o período prioritário de atuação no combate ao excesso de peso e obesidade. ${ }^{21}$ Como referem de Onis $\mathrm{M}$ et al, ${ }^{22}$ uma vez estabelecida a obesidade na criança, é muito difícil reverter o problema, sendo a monitorização da sua prevalência um ponto-chave para a definição e avaliação do impacto de políticas e estratégias desenvolvidas a este nível. ${ }^{22-24}$ Para além disso, a OMS reforça a necessidade de uma abordagem multissectorial que envolva o setor agroalimentar, a educação, os governos locais e a saúde, de forma concertada. ${ }^{25}$

Os governos locais, particularmente, as autarquias, pelas áreas de ação que lhe competem, dispõem de meios que permitem conhecer e combater a obesidade infantil atuando em vários dos seus principais determinantes de forma concertada, nomeadamente, nos ambientais, nos culturais e nos socioeconómicos. ${ }^{26,27}$

Atualmente, Vila Nova de Gaia é o terceiro município mais populoso de Portugal, com um total de 302295 habitantes. Destes, 46641 têm idades compreendidas entre os 0 e os 14 anos. $^{28}$

Tal como todos os outros em Portugal, este município é responsável, ao abrigo do Lei 75/2013 de 12 de setembro, ${ }^{26}$ pela gestão dos níveis de ensino pré-escolar (PE) e primeiro ciclo do ensino básico (1CEB), exceto no que respeita às questões pedagógicas e de gestão do pessoal docente, tendo como sua responsabilidade a gestão das refeições escolares. Estes níveis de ensino estão organizados por Agrupamentos de Escolas.

Ao longo dos últimos anos, vários estudos à escala nacional e/ou regional têm sido conduzidos, no sentido de tentar avaliar a prevalência de excesso de peso (pré-obesidade e obesidade) na população infantil portuguesa. ${ }^{14,29-32}$ De uma forma geral, verifica-se que a prevalência de pré-obesidade e obesidade infantil é elevada, ${ }^{14,29-32}$ podendo ser superior a $30 \%{ }^{32}$ o valor de crianças portuguesas com excesso de peso. De facto, o 'Programa Nacional para a Promoção da Alimentação Saudável', ${ }^{33}$ criado em 2012 pela Direção-Geral da Saúde, tem como um dos seus indicadores, controlar a prevalência de excesso de peso na população infantil e escolar, limitando o crescimento a zero, até 2016. ${ }^{33}$ Já no Plano Nacional de Saúde, na sua edição revista e estendida até 2020 , uma das suas quatro metas fundamentais é o não crescimento da obesidade infantil. ${ }^{34}$

Assim, considerando as metas nacionais definidas, este trabalho de investigação pretende caracterizar a atual situação, relativamente à prevalência de pré-obesidade e obesidade nas crianças do concelho de Vila Nova de Gaia. Pretende ainda demonstrar que é possível realizar uma avaliação da pré-obesidade e obesidade infantil tendo por base os serviços autárquicos, para, a partir daqui, definir estratégias de intervenção que utilizem e potenciem as competências e meios à disposição dos municípios.

\section{MATERIAL E MÉTODOS}

Este é um estudo observacional, descritivo e transversal promovido pelo Departamento de Educação da Câmara Municipal de Vila Nova de Gaia e realizado nos estabelecimentos de ensino PE e 1CEB da rede pública, que decorreu 
durante o ano letivo 2013/14.

Em Vila Nova de Gaia, no período de estudo, existiam 87 estabelecimentos de ensino do PE e 100 estabelecimentos de ensino do 1CEB, reunidos em 14 Agrupamentos de Escolas, perfazendo um total de 13951 alunos (3 527 PE e 10424 1CEB).

Todos os 13951 alunos inscritos nos 187 estabelecimentos de ensino foram convidados a participar. Foi enviado aos encarregados de educação uma explicação do objetivo do estudo, com indicação de quem o estava a desenvolver e seus contactos, dados a recolher e destino dos resultados, garantindo o anonimato dos mesmos, informando que a participação tinha carácter voluntário não se vislumbrando qualquer prejuízo no bem-estar dos seus educandos. Só foram avaliados os alunos cujos encarregados de educação expressaram a sua autorização, assinando o termo de consentimento informado acima descrito, o qual foi elaborado com base nas International Ethical Guidelines for Biomedical Research Involving Humam Subjects, ${ }^{35}$ como proposto na metodologia do World Health Organization (WHO) European Childhood Obesity Surveillance Iniciative $(\mathrm{COSI}),{ }^{32,36}$ que serviu de referência à construção metodológica do presente estudo.

Para além do consentimento informado dos encarregados de educação, o estudo foi enviado para avaliação pelas Direções dos Agrupamentos de Escolas e Coordenações de cada estabelecimento de ensino, os quais autorizaram e facilitaram, sem exceção, a realização desta investigação.

Neste estudo participaram 8974 indivíduos (2 697 alunos do PE e 6277 alunos do 1 CEB) com idades compreendidas entre os três e os 13 anos de idade.

A diferença entre o número total de alunos participantes e o total da amostra inicial, da qual foram excluídos os que apresentavam incapacidade física para a realização da avaliação antropométrica, corresponde aos alunos não autorizados ou alunos que faltaram à escola no dia da avaliação.

Apesar dos resultados individuais não terem sido comunicados a cada aluno, houve lugar à devida informação sempre que algum encarregado de educação manifestou esse interesse.

\section{Variáveis}

Dos dados recolhidos, fazem parte as informações do aluno disponibilizadas pela escola: data de nascimento, género e morada e as informações obtidas através das avaliações antropométricas: peso, altura e data da avaliação.

\section{Recolha e tratamento de dados}

As avaliações antropométricas foram realizadas por colaboradores treinados de acordo com a metodologia preconizada pela OMS. ${ }^{37}$ No ano letivo, alvo da presente investigação o treino decorreu no mês de dezembro, consistindo numa abordagem teórica ao tema, explicação do trabalho e seu objetivo (45 minutos), seguida pela explicação e padronização do procedimento de avaliação antropométrica e treino prático entre os colaboradores (120 minutos).
Antes de se proceder às medições, cada criança foi questionada verbalmente no sentido de autorizar o procedimento, tendo todas as crianças dado o seu aval.

O peso foi avaliado utilizando uma balança eletrónica digital idêntica, registando-se o valor até ao hectograma mais próximo $(0,1 \mathrm{~kg})$. Com o objetivo de aferir as 85 balanças utilizadas, uma delas foi calibrada pelo serviço de metrologia da Câmara Municipal de Vila Nova de Gaia nas gamas de leitura $10 \mathrm{~kg}, 20 \mathrm{~kg}, 30 \mathrm{~kg}, 40 \mathrm{~kg}, 50 \mathrm{~kg}, 60 \mathrm{~kg}$, $70 \mathrm{~kg}$ e $80 \mathrm{~kg}$. As restantes foram verificadas realizando três avaliações comparativas com a balança calibrada. As diferenças encontradas foram registadas e consideradas aquando do tratamento dos dados de cada escola.

A estatura foi avaliada utilizando uma fita métrica não extensível fixada a uma parede lisa e vertical, estando a cabeça posicionada para que o olhar se mantivesse horizontal, de acordo com o plano de Frankfort ${ }^{38}$ e o queixo segurado pelos dedos indicador e polegar do avaliador, usando como craveira um objeto plano retangular colocado a $90^{\circ}$. Registou-se o valor até ao milímetro mais próximo $(0,1 \mathrm{~cm})$. Esta avaliação foi realizada duas vezes, sendo considerado o valor médio das duas medições e os resultados registados em suporte informático.

Com o objetivo de validar as medições efetuadas, foram selecionadas, aleatoriamente, 10 escolas e procedeu-se à repetição das mesmas, desta vez realizada pelas nutricionistas da Câmara. Para além destas, repetiram-se as avaliações em mais três escolas. Esta repetição de procedimentos resultou de uma primeira verificação e limpeza de todas as medições efetuadas, realizada a posteriori e da verificação de registos pouco comuns em três escolas, tendo em atenção os valores médios obtidos.

A avaliação antropométrica decorreu entre 24 de fevereiro e 6 de abril de 2014 e as crianças tinham vestido apenas uma peça superior e outra inferior (exceto roupa interior). Para a definição do período de avaliação, foi considerado um intervalo máximo de dois meses entre o início e o fim dos procedimentos e ainda, o seu início ocorrer, pelo menos, duas semanas após o começo do período letivo. ${ }^{36}$

Os dados avaliados foram introduzidos no software $\mathrm{An}$ thro ${ }^{\circledR}$ (para os alunos com idade até aos 60 meses) e no software AnthroPlus ${ }^{\circledR 39}$ (para os alunos com idade superior a 60 meses), permitindo obter, para cada indivíduo, o indice de massa corporal (IMC), percentil de IMC ajustado à idade e Z-score do IMC para a idade.

\section{Referenciais escolhidos para a classificação do estado nutricional}

O IMC foi o indicador antropométrico escolhido para a avaliação da prevalência de pré-obesidade e obesidade infantil, sendo o preditor internacional de obesidade adotado e aceite como medida indireta de adiposidade em crianças. ${ }^{40}$

Para a classificação do estado nutricional foram considerados os percentis ou z-scores do IMC para a idade de acordo com os critérios e pontos de corte definidos pela OMS, ${ }^{22,41,42}$ pelo Centers for Disease Control and Prevention 
$(\mathrm{CDC})^{43}$ e pela International Obesity Task Force (IOTF), ${ }^{42,44}$ conforme apresentado na Tabela 1.

Para o tratamento e posterior análise dos dados foi feita uma abordagem geral seguida da divisão da amostra em dois subgrupos (< que cinco anos de idade e entre 5-13 anos de idade). Esta divisão deve-se à necessidade de avaliar separadamente estes dois grupos de acordo com o critério da OMS. ${ }^{41,45}$

$\mathrm{Na}$ análise geral da amostra, foi preciso uniformizar a linguagem para os diferentes critérios, particularmente para idades inferiores a cinco anos, tendo-se optado por incluir as crianças classificadas como apresentando 'risco de excesso de peso' no escalão da 'pré-obesidade'.

\section{Tratamento e análise dos dados}

Foi realizada uma análise estatística descritiva dos resultados com recurso ao programa Statistical Package for Social Sciences (SPSS ${ }^{\circledR}$ ) versão 22.0.

O tratamento estatístico considerou as médias e desvios-padrão das variáveis quantitativas e frequências das variáveis categóricas. As diferenças nas variáveis categóricas foram comparadas através do teste qui-quadrado. As variáveis quantitativas foram comparadas por teste $t$ de Student. O nível de significância foi considerado abaixo de 0,05 .

\section{RESULTADOS}

\section{Caracterização da amostra}

Este estudo envolveu 8974 crianças com idades compreendidas entre os três e os 13 anos, oriundas de 93 dos 113 estabelecimentos de ensino pré-escolar e primeiro ciclo do ensino básico que constituíam os 14 Agrupamentos de Escolas de Vila Nova de Gaia.

$\mathrm{Na}$ Tabela 2 estão apresentadas as características antropométricas da população em estudo, por género. Pela sua análise pudemos constatar a existência de uma grande homogeneidade na distribuição da amostra por sexos, seja na amostra total $(51,30 \%$ sexo masculino e $48,70 \%$ sexo feminino), seja no subgrupo com idade inferior a cinco anos (48,96\% sexo masculino e $51,04 \%$ sexo feminino) ou no subgrupo que compreendeu indivíduos com idades entre os cinco os 13 anos (51,74\% sexo masculino e $48,26 \%$ sexo feminino). Relativamente ao peso e à altura, na generalidade, mesmo mantendo-se valores próximos para ambos os sexos, pudemos verificar que os rapazes apresentaram valores mais elevados (peso: rapazes - 27,58 kg $\pm 8,51$ e raparigas $-27,28 \mathrm{~kg} \pm 9,02 ; p=0,096$; altura: rapazes $-125,23 \mathrm{~cm} \pm 12,66$ e raparigas $-123,81 \pm 13,35$; $p<0,001)$, o que se inverteu quando analisámos o IMC (rapazes $-17,22 \pm 2,77$ e raparigas $-17,34 \pm 2,92 ; p=$ 0,041). Em todas as situações, as meninas apresentaram um maior grau de variação de valores. Quando fizemos a

Tabela 1 - Classificação do IMC de acordo com três critérios: IOTF, CDC e OMS (2006 e 2007)

\begin{tabular}{|c|c|c|c|c|}
\hline & Percentil IMC (P) & Classificação & & \\
\hline \multirow{5}{*}{$\begin{array}{l}\text { IOTF } \\
2 \text { - } 18 \text { anos } \\
\text { idade }^{44}\end{array}$} & $p<15,5$ & Magreza & & \\
\hline & $15,5 \leq p<90,5$ & Peso normal & & \\
\hline & $p \geq 90,5$ & $\begin{array}{l}\text { Excesso de peso } \\
\text { (inclui obesidade) }\end{array}$ & & \\
\hline & $90,5 \leq p<98,9$ & Pré-obesidade & & \\
\hline & $p \geq 98,9$ & Obesidade & & \\
\hline \multirow{7}{*}{$\begin{array}{l}\text { CDC } 2000 \\
2 \text { - } 18 \text { anos } \\
\text { idade }^{43}\end{array}$} & $p<5$ & Baixo peso & & \\
\hline & $5 \leq p<85$ & Peso normal & & \\
\hline & $p \geq 85$ & $\begin{array}{l}\text { Excesso de peso } \\
\text { (inclui obesidade) }\end{array}$ & & \\
\hline & $85 \leq p<95$ & Pré-obesidade & & \\
\hline & $p \geq 95$ & Obesidade & & \\
\hline & \multicolumn{2}{|c|}{$<5$ anos idade (OMS, 2006) } & \multicolumn{2}{|c|}{5 a 19 anos idade (OMS, 2007) } \\
\hline & Z-score (Zsc) de IMC & Classificação & Z-score (Zsc) de IMC & Classificação \\
\hline \multirow{6}{*}{$\begin{array}{l}\text { OMS } 2006^{41} \mathrm{e} \\
2007^{45}\end{array}$} & $Z s c<-2$ & Magreza & $Z s c<-2$ & Magreza \\
\hline & $-2 \leq Z s c \leq+1$ & Peso normal & $-2 \leq Z s c \leq+1$ & Peso normal \\
\hline & $Z s c>+1$ & $\begin{array}{l}\text { Excesso de peso } \\
\text { (inclui obesidade) }\end{array}$ & $Z s c>+1$ & $\begin{array}{l}\text { Excesso de peso } \\
\text { (inclui obesidade) }\end{array}$ \\
\hline & $+1<Z s c \leq+2$ & Risco excesso peso & $+1<Z s c \leq+2$ & Pré-obesidade \\
\hline & $+2<Z s c \leq+3$ & Pré-obesidade & $Z s c>+2$ & Obesidade \\
\hline & $Z s c>+3$ & Obesidade & & \\
\hline
\end{tabular}

IMC: índice de massa corporal; OMS: Organização Mundial de Saúde; CDC: Centre for Diseases Control and Prevention; IOTF: International Obesity Task Force 
mesma análise para os dois subgrupos verificámos que para os indivíduos entre os cinco e os 13 anos de idade se manteve a tendência descrita para o geral - rapazes mais pesados, mais altos e com menor IMC relativamente às raparigas, mas no subgrupo dos indivíduos com idade inferior a cinco anos constatámos que as meninas apresentavam menor IMC $(16,27 \pm 1,97)$, comparativamente aos rapazes.

\section{Prevalência de excesso de peso. Pré-obesidade e obe- sidade.}

$\mathrm{Na}$ Tabela 3 estão apresentadas as prevalências, por sexo, de acordo com os 3 critérios escolhidos (CDC, IOTF e OMS). Constatámos uma menor prevalência de excesso de peso (pré-obesidade + obesidade), quando utilizámos o critério da IOTF $(31,85 \%)$, e, muito semelhante para os critérios do CDC e da OMS, 37,54\% e 37,44\%, respetivamente. Relativamente à distribuição por sexos, encontrámos valores de excesso de peso superiores para os meninos. No entanto, ao desdobrarmos o excesso de peso em pré-obesidade (PO) e obesidade $(\mathrm{O})$, verificámos que para a classificação feita pela OMS e pelo IOTF a prevalência de excesso de peso teve uma maior contribuição dos indivíduos pré-obesos, $22,16 \%$ e $19,69 \%$ respetivamente, no caso da classificação prevista pelo CDC foram os indivíduos obesos que mais contribuíram para a taxa de excesso de peso encontrada $(22,14 \%)$.

Nas Tabelas 4 e 5 apresentamos a classificação da amostra, quanto ao IMC, agora dividida pelo dois subgrupos de idade (< que cinco anos e entre cinco e 13 anos). Verificámos que a prevalência de excesso de peso (incluindo a obesidade) se apresentou sempre acima dos 30\%, com exceção dos indivíduos com menos de cinco anos de idade pelo critério da IOTF. Quando se observou cada um dos parâmetros do excesso de peso, separadamente, constatamos que para o subgrupo com idade inferior a cinco anos, a percentagem de crianças consideradas obesas pela OMS $(2,52 \%)$ foi inferior à considerada pela IOTF $(8,44 \%)$, aumentado ainda mais a diferença quando se comparou com o CDC (18,59\%). Para as crianças com idades entre os cinco e os 13 anos, o critério do CDC continuou a ser o que apresentou maior percentagem de indivíduos obesos, estando sempre acima dos $20 \%$, independentemente do sexo. Já para os critérios da OMS e da IOTF, verificámos uma inversão do observado no subgrupo da idade inferior a cinco anos, tendo sido verificada uma percentagem superior de obesos pelo critério da OMS.

$\mathrm{Na}$ Tabela 6 apresentamos de forma resumida a distribuição da prevalência de pré-obesidade e obesidade pelos 14 Agrupamentos de escolas do Concelho de Vila Nova de Gaia. Pela sua análise pudemos verificar existirem diferenças consideráveis na distribuição geográfica do problema, sendo o Agrupamento Soares dos Reis o que a apresentou a maior a prevalência $(54,41 \%)$ e o Agrupamento Gaia Nascente o que apresentou a menor $(32,89 \%)$, sendo as diferenças entre eles estatisticamente significativas $(p=0,003)$.

\section{DISCUSSÃO}

Alguns autores vêm com alguma relutância a utilização do IMC, isoladamente, como o indicador antropométrico preditivo de excesso de peso. ${ }^{5}$ No entanto, as suas vantagens, no que respeita à facilidade de aplicação, ao baixo custo associado e ao facto de se tratar de uma avaliação não invasiva, somadas às dificuldades inerentes a outros métodos de avaliação da quantidade de gordura corporal e à falta de referências que permitam a sua interpretação, fazem do IMC ajustado para a idade, o melhor indicador para definir a pré-obesidade e a obesidade infantil, ${ }^{22}$ razão pela qual optamos pela sua utilização nesta investigação.

Atualmente, existem vários critérios para o diagnóstico de excesso de peso na população infantil (pré-obesidade e obesidade). ${ }^{12,29}$ A European Childhood Obesity Group ${ }^{46}$ bem como a International Pediatric Association, ${ }^{47}$ recomendam que se usem os pontos de corte definidos pela OMS para crianças dos 0 aos cinco anos (OMS 2006) (11 $^{4}$ e dos cinco aos 19 anos e idade (OMS 2007), ${ }^{45}$ no sentido de

Tabela 2 - Caracterização antropométrica da amostra em estudo

\begin{tabular}{|c|c|c|c|c|c|c|c|}
\hline & & $\mathbf{N}$ & $\begin{array}{c}\text { Idade } \\
\text { (anos) } \\
\text { média } \pm d p\end{array}$ & $\begin{array}{c}\text { Peso } \\
\text { (kg) } \\
\text { média } \pm d p\end{array}$ & $\begin{array}{c}\text { Altura } \\
\text { (cm) } \\
\text { média } \pm d p\end{array}$ & $\begin{array}{c}\text { IMC } \\
\text { média } \pm d p\end{array}$ & $\begin{array}{l}\text { Percentil de IMC } \\
\text { para a Idade (\%) } \\
\text { média } \pm d p\end{array}$ \\
\hline \multirow{3}{*}{ Geral } & Total & $8974(100)$ & $6,78 \pm 1,97$ & $27,44 \pm 8,76$ & $124,54 \pm 13,02$ & $17,28 \pm 2,84$ & $68,88 \pm 29,95$ \\
\hline & Masculino & $4606(51,30)$ & $6,81 \pm 1,96$ & $27,58 \pm 8,51$ & $125,23 \pm 12,66$ & $17,22 \pm 2,77$ & $66,95 \pm 30,19$ \\
\hline & Feminino & $4368(48,70)$ & $6,75 \pm 1,97$ & $27,28 \pm 9,02$ & $123,81 \pm 13,35$ & $17,34 \pm 2,92$ & $65,81 \pm 29,70$ \\
\hline \multirow{3}{*}{$\begin{array}{l}3-4 \\
\text { anos }\end{array}$} & Total & 1350 & $3,74 \pm 0,44$ & $18,32 \pm 3,10$ & $105,84 \pm 6,18$ & $16,29 \pm 1,86$ & $65,13 \pm 29,43$ \\
\hline & Masculino & $661(48,96)$ & $3,75 \pm 0,44$ & $18,63 \pm 2,93$ & $106,72 \pm 6,20$ & $16,31 \pm 1,72$ & $66,17 \pm 28,93$ \\
\hline & Feminino & $689(51,04)$ & $3,73 \pm 0,45$ & $18,03 \pm 3,24$ & $105,00 \pm 6,04$ & $16,27 \pm 1,97$ & $64,13 \pm 29,89$ \\
\hline \multirow{3}{*}{$\begin{array}{l}5-13 \\
\text { anos }\end{array}$} & Total & 7624 & $7,32 \pm 1,61$ & $29,05 \pm 8,45$ & $127,85 \pm 10,95$ & $17,46 \pm 2,95$ & $66,01 \pm 30,04$ \\
\hline & Masculino & $3945(51,74)$ & $7,33 \pm 1,61$ & $29,09 \pm 8,21$ & $128,34 \pm 10,66$ & $17,37 \pm 2,88$ & $65,91 \pm 30,40$ \\
\hline & Feminino & $3679(48,26)$ & $7,32 \pm 1,60$ & $29,01 \pm 8,69$ & $127,33 \pm 11,23$ & $17,54 \pm 3,03$ & $66,13 \pm 29,65$ \\
\hline
\end{tabular}

IMC: índice de massa corporal 
Tabela 3 - Classificação da amostra de acordo com o IMC, para os 3 critérios (OMS, CDC e IOTF)

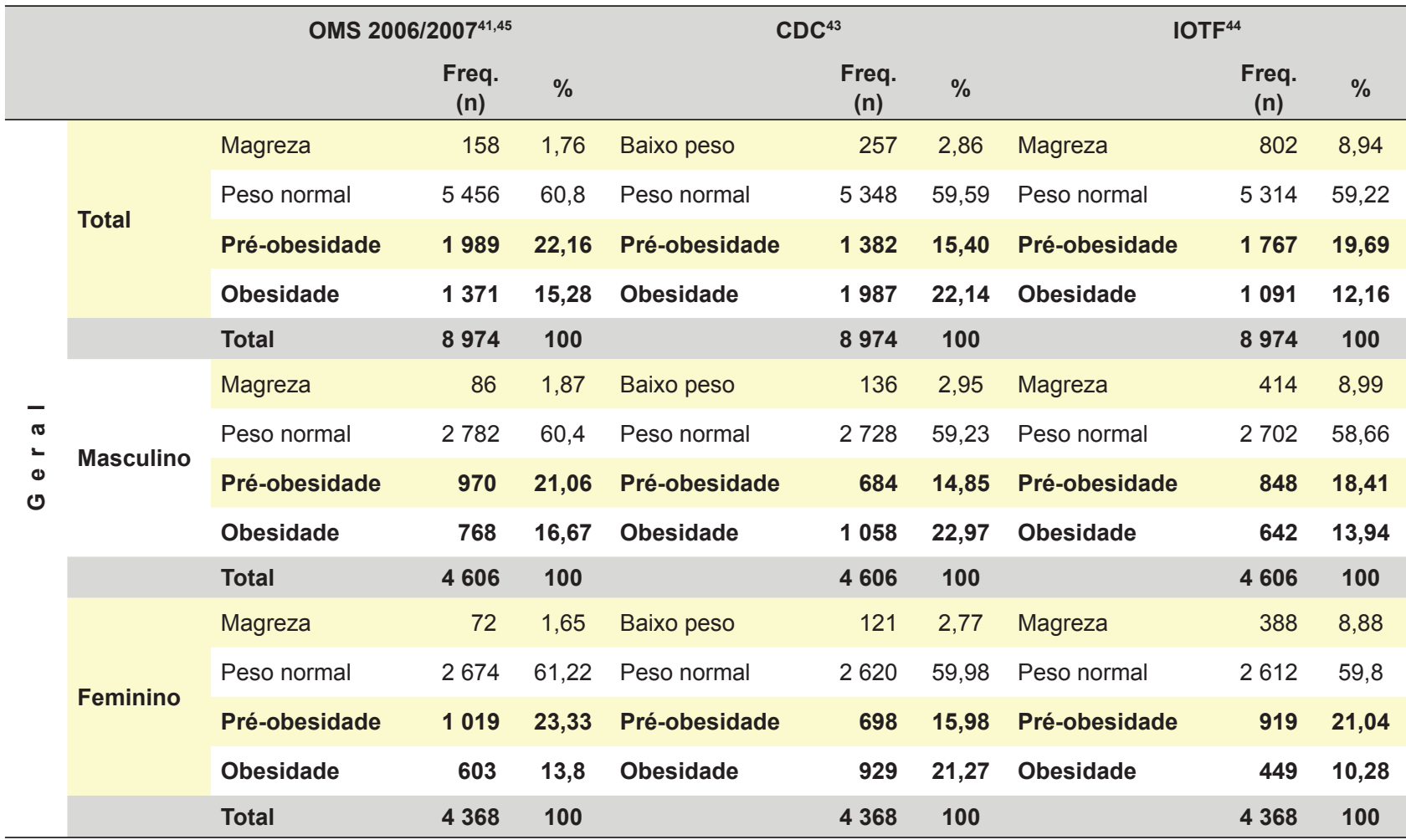

IMC: índice de massa corporal; OMS: Organização Mundial de Saúde; CDC: Centre for Diseases Control and Prevention; IOTF: International Obesity Task Force

Tabela 4 - Classificação dos indivíduos com idade inferior a 5 anos, de acordo com o IMC, para os 3 critérios (WHO, CDC e IOTF)

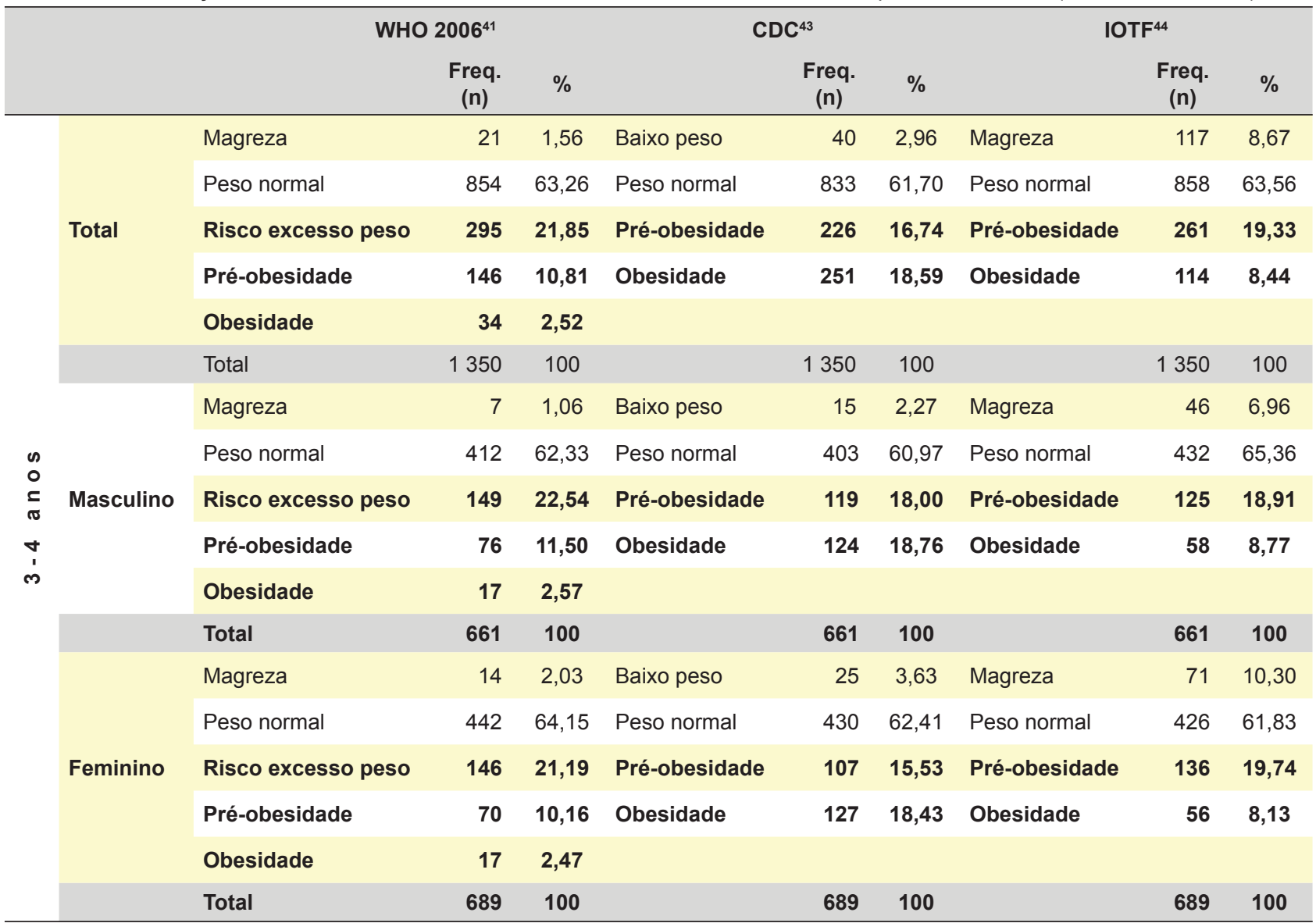

IMC: índice de massa corporal; OMS: Organização Mundial de Saúde; CDC: Centre for Diseases Control and Prevention; IOTF: International Obesity Task Force 
harmonizar protocolos e melhorar a qualidade na comparação entre os diferentes estudos e, consequentemente, melhorar a qualidade de pesquisa e vigilância acerca deste problema. ${ }^{46,47}$ No entanto, continuam a surgir muitos trabaIhos em que os pontos de corte utilizados são os previstos pelo CDC e pela IOTF, ${ }^{14,30,48,49}$ o que levou a que neste estudo fizéssemos uma avaliação de acordo com os três critérios, aumentando, assim, a possibilidade de comparação com os resultados de outros trabalhos.

Relativamente aos resultados encontrados verificamos que eles se encontram em concordância com outros estudos nacionais e internacionais em que a prevalência de excesso de peso, se apresenta a rondar os $30 \%$. $^{14,18,30,50}$

São poucos os trabalhos nacionais e/ou internacionais que apresentem uma gama de idades desde a idade pré-escolar (três anos) até aos 10 anos (fim do primeiro ciclo de ensino básico). A nível Europeu, no âmbito do projeto Identification and prevention of Dietary - and lifestyle - induced health Effects In Children and infantS (IDEFICS) ${ }^{51}$ que estudou crianças com menos de 10 anos de idade, tal como noutros trabalhos, verificou-se uma maior prevalência de excesso de peso nos países do sul da Europa ${ }^{52} \mathrm{com}$ a Itália a liderar com $42,4 \% .^{51}$ Porém, o nosso valor apresenta-se ligeiramente superior ao reportado por Nogueira $\mathrm{H}$ et $\mathrm{al}^{53}$ que atribuíram $26,8 \%$ à prevalência de excesso de peso $(\mathrm{PO}+\mathrm{O}) . .^{53}$

A nível internacional, a Organização para a Cooperação e Desenvolvimento Económico (OCDE) apresentou em 2011, para a prevalência de excesso de peso, valores de $21,4 \%$ para as raparigas e $22,9 \%$ para os rapazes. Nes- se mesmo estudo, Portugal já se encontrava ligeiramente acima da média, com valores de $21,6 \%$ para as raparigas e $23,5 \%$ para os rapazes, ${ }^{54}$ valores manifestamente inferiores aos encontrados na presente investigação, achado que poderá estar relacionado com diferenças tanto a nível da amostra como com a distância temporal de recolha dos dados.

Conhecida a dificuldade em analisar comparativamente resultados de estudos de prevalência de excesso de peso, quer pela reduzida quantidade de publicações quer pela falta de uniformidade da metodologia, quando passamos a analisar o subgrupo de crianças com idade inferior a 5 anos, esta dificuldade acentua-se..$^{51}$ Assim, os resultados encontrados para o subgrupo de crianças com menos de 5 anos de idade, de acordo com o critério da IOTF apresentam prevalência de pré-obesidade e obesidade superiores a outros estudos. ${ }^{52,55}$ Ao comparar as prevalências pelo critério do CDC, os estudos de Lourenço $M$ et al ${ }^{49}$ e Rito $A^{55}$ apesar de apresentarem resultados ligeiramente inferiores, podemos dizer que estão em concordância com o nosso. Relativamente ao critério previsto pela OMS, o presente trabalho apresenta uma prevalência de excesso de peso superior ao estudo de Rito A. ${ }^{55}$

Para a análise dos resultados encontrados para o subgrupo dos cinco aos 13 anos de idade o principal estudo de comparação é o COSI. À semelhança do descrito por Wijnhoven $\mathrm{T}$ et $\mathrm{al}^{18} \mathrm{e}$ por Rito $\mathrm{A}$ et $a l,{ }^{56}$ as prevalências de pré-obesidade, segundo o critério da OMS 2007 são sempre mais elevadas, independentemente do sexo. No entanto, relativamente à prevalência de obesidade, o

Tabela 5 - Classificação dos indivíduos com idade entre os 5 e os 13 a 5 anos, de acordo com o IMC, para os 3 critérios (WHO, CDC e IOTF)

\begin{tabular}{|c|c|c|c|c|c|c|c|c|c|c|}
\hline & & \multicolumn{3}{|c|}{ WHO $2007^{45}$} & \multicolumn{3}{|c|}{$\mathrm{CDC}^{43}$} & \multicolumn{3}{|c|}{ IOTF 44} \\
\hline & & & $\begin{array}{l}\text { Freq. } \\
\text { (n) }\end{array}$ & $\%$ & & $\begin{array}{l}\text { Freq. } \\
\text { (n) }\end{array}$ & $\%$ & & $\begin{array}{l}\text { Freq. } \\
\text { (n) }\end{array}$ & $\%$ \\
\hline \multirow{15}{*}{$\begin{array}{l}0 \\
0 \\
c \\
\sigma \\
0 \\
m \\
\text { n' }\end{array}$} & \multirow{5}{*}{ Total } & Magreza & 137 & 1,80 & Baixo peso & 217 & 2,85 & Magreza & 658 & 8,63 \\
\hline & & Peso normal & 4602 & 60,36 & Peso normal & 4515 & 59,22 & Peso normal & 4569 & 59,93 \\
\hline & & Pré-obesidade & 1548 & 20,30 & Pré-obesidade & 1156 & 15,16 & Pré-obesidade & 1490 & 19,54 \\
\hline & & Obesidade & 1337 & 17,54 & Obesidade & 1736 & 22,77 & Obesidade & 907 & 11,90 \\
\hline & & Total & 7624 & 100 & & 7624 & 100 & & 7624 & 100 \\
\hline & \multirow{5}{*}{ Masculino } & Magreza & 79 & 2,00 & Baixo peso & 121 & 3,07 & Magreza & 341 & 8,64 \\
\hline & & Peso normal & 2370 & 60,08 & Peso normal & 2325 & 58,94 & Peso normal & 2383 & 60,41 \\
\hline & & Pré-obesidade & 745 & 18,88 & Pré-obesidade & 565 & 14,32 & Pré-obesidade & 707 & 17,92 \\
\hline & & Obesidade & 751 & 19,04 & Obesidade & 934 & 23,68 & Obesidade & 514 & 13,03 \\
\hline & & Total & 3945 & 100 & & 3945 & 100 & & 3945 & 100 \\
\hline & \multirow{5}{*}{ Feminino } & Magreza & 58 & 1,58 & Baixo peso & 96 & 2,61 & Magreza & 317 & 8,62 \\
\hline & & Peso normal & 2232 & 60,67 & Peso normal & 2190 & 59,53 & Peso normal & 2186 & 59,42 \\
\hline & & Pré-obesidade & 803 & 21,83 & Pré-obesidade & 591 & 16,06 & Pré-obesidade & 783 & 21,28 \\
\hline & & Obesidade & 586 & 15,93 & Obesidade & 802 & 21,80 & Obesidade & 393 & 10,68 \\
\hline & & Total & 3679 & 100 & & 3679 & 100 & & 3679 & 100 \\
\hline
\end{tabular}

IMC: índice de massa corporal; OMS: Organização Mundial de Saúde; CDC: Centre for Diseases Control and Prevention; IOTF: International Obesity Task Force 
Tabela 6 - Distribuição da prevalência de excesso de peso (incluindo a obesidade) pelos 14 Agrupamentos de escolas, de acordo com o critério

\begin{tabular}{|c|c|c|c|c|c|c|}
\hline & $\begin{array}{l}\text { Agrupamento } \\
\text { António Sérgio } \\
\qquad n=329\end{array}$ & $\begin{array}{c}\text { Agrupamento } \\
\text { Canelas } \\
n=1112\end{array}$ & $\begin{array}{l}\text { Agrupamento } \\
\text { Carvalhos } \\
n=824\end{array}$ & $\begin{array}{l}\text { Agrupamento } \\
\text { D. Pedro } \\
n=852\end{array}$ & $\begin{array}{l}\text { Agrupamento } \\
\text { Diogo Macedo } \\
n=273\end{array}$ & $\begin{array}{c}\begin{array}{l}\text { Agrupamento } \\
\text { Dr. Costa Mato } \\
n=932\end{array}\end{array}$ \\
\hline Percentagem de crian & & & & & & \\
\hline $\begin{array}{l}\text { excesso de peso } \\
\text { (incluindo obesidade) } \\
\text { Critério OMS }\end{array}$ & $\begin{array}{c}40,42 \% \\
(n=133)\end{array}$ & $\begin{array}{c}39,83 \% \\
(n=443)\end{array}$ & $\begin{array}{c}45,02 \% \\
(n=371)\end{array}$ & $\begin{array}{c}37,68 \% \\
(n=321)\end{array}$ & $\begin{array}{l}36,26 \% \\
(n=99)\end{array}$ & $\begin{array}{c}33,26 \% \\
(n=310)\end{array}$ \\
\hline
\end{tabular}

OMS: Organização Mundial de Saúde

critério do CDC mostra-se o mais sensível nesta avaliação comparativamente com os dois autores citados. Já Padez $\mathrm{C}$ et al ${ }^{30}$ encontraram valores muito concordantes com os do presente estudo para a prevalência de pré-obesidade $(20,3 \%$ e $19,54 \%)$ e de obesidade $(11,3 \%$ e $11,90 \%)$. Na investigação de Cordinhã $\mathrm{AC}$ et $\mathrm{a}^{48}$ foram achados valores de excesso de peso $(\mathrm{PO}+\mathrm{O})$ inferiores aos apresentados nesta investigação com exceção da percentagem de raparigas com pré-obesidade $(20,3 \%)$ que no nosso trabalho foi de $16,06 \%$. Ainda em comparação com este subgrupo, Pedrosa $\mathrm{C}$ et al, ${ }^{31}$ para uma amostra com idades entre os sete e os nove anos encontraram prevalências inferiores ao presente estudo $(22,77 \%)$, nomeadamente, no que se refere à prevalência de obesidade pelo critério do CDC (14,0\%).

As diferenças evidenciadas neste estudo poderão dever-se ao facto de a amostra corresponder à quase totalidade da população em estudo, bem como ao intervalo alargado de idades abrangido, uma vez que a maioria dos estudos o restringe a um ou dois anos de idade a população avaliada.

A investigação aqui apresentada diz respeito ao ano letivo 2013/2014, no entanto insere-se num trabalho de continuidade iniciado em 2008/2009 para o ensino pré-escolar. No presente momento, abril de 2015, encontra-se na fase de recolha de dados relativa a 2014/2015. É uma monitorização que pretendemos manter com caráter anual. Pelo facto de ser um trabalho desenvolvido pela própria autarquia, que usa como amostra a totalidade da população escolar dos níveis de ensino abrangidos pela sua tutela, garantindo uma excelente representatividade do Concelho, nas suas diferentes realidades, quer geográficas quer sociodemográficas, o mesmo reveste-se de extrema importância como ferramenta decisora para a tomada de decisões políticas e executivas no que respeita a várias competências do poder local, nomeadamente, na área da saúde, educação, ação social, desporto, infraestruturas e licenciamento comercial. ${ }^{26} \mathrm{Apesar}$ da avaliação da distribuição do excesso de peso pelo concelho não ser o principal objetivo deste documento, constatamos existirem diferenças consideráveis entre Agrupamentos de Escolas, o que permitirá futuramente relacionar as características sociodemográficas e de tecido urbano local com as prevalências de excesso de peso encontradas.

Trabalhos como este vão ao encontro do preconizado no Plano de Ação Europeu para a Alimentação e Nutrição 2015-202025 bem como na Declaração de Viena para a Nu- trição e Doenças Crónicas no Contexto da Saúde 2020,57 que reconhecem o papel das Autarquias na promoção de uma alimentação saudável, encorajamento da prática de atividade física e prevenção da obesidade infantil, ${ }^{57}$ sendo para tal necessário conhecerem a realidade local que lhes permita tomar decisões ajustadas à mesma.

\section{CONCLUSÃO}

Independentemente do sistema de classificação utilizado ou do subgrupo analisado, a prevalência de excesso de peso $(\mathrm{PO}+\mathrm{O})$ encontrada é muito elevada no concelho de Vila Nova de Gaia para crianças entre os três e os 13 anos de idade, apresentando quase sempre valores superiores aos estudos nacionais existentes e comparáveis.

Os resultados obtidos revestem-se de grande importância. Embora fosse já conhecida a elevada prevalência de excesso de peso infantil em Portugal, esta nova caracterização permite agora identificar com maior exatidão o problema a nível local (prevalência de $22,16 \%$ de pré-obesidade e $15,28 \%$ de obesidade para as crianças que frequentam o ensino pré-escolar e o primeiro ciclo do ensino básico da rede pública do concelho de Vila Nova de Gaia). Os dados revelam ainda uma grande variação geográfica nas diferentes escolas do concelho, inseridas em diferentes realidades socioeconómicas e urbanas.

Estes achados e a possibilidade desta monitorização de base autárquica continuar, permitirão agora à Autarquia planear, implementar e avaliar os efeitos de diferentes estratégias no combate a este grave problema, nomeadamente no controlo da oferta alimentar nas escolas, bem como no controlo do licenciamento de estabelecimentos de restauração na proximidade das mesmas; no incentivo e influência na prática de atividade física, seja pela disponibilização desta disciplina nas atividades de enriquecimento curricular seja pela responsabilidade e capacidade que the está atribuída na construção e manutenção de infraestruturas facilitadoras da prática de atividade física; e ainda pela capacidade de controlo e modificação de alguns determinantes sociais, geográficos e demográficos.

\section{AGRADECIMENTOS}

Agradecemos a todas as famílias que participaram no estudo, bem como a todos os professores, assistentes técnicos e assistentes operacionais de Vila Nova de Gaia que participaram na recolha e avaliação dos dados antropométricos. 


\begin{tabular}{|c|c|c|c|c|c|c|c|}
\hline $\begin{array}{l}\text { Agrupamento } \\
\text { Escultor } \\
\text { Fernandes Sá } \\
\quad n=434\end{array}$ & $\begin{array}{l}\text { Agrupamento } \\
\text { Gaia Nascente } \\
\qquad n=152\end{array}$ & $\begin{array}{l}\text { Agrupamento } \\
\text { Júlio Dinis } \\
n=693\end{array}$ & $\begin{array}{l}\text { Agrupamento } \\
\text { Madalena } \\
n=254\end{array}$ & $\begin{array}{c}\text { Agrupamento } \\
\text { Soares Reis } \\
n=68\end{array}$ & $\begin{array}{c}\text { Agrupamento } \\
\text { Sophia Mello } \\
\text { Breyner } \\
n=1281\end{array}$ & $\begin{array}{l}\text { Agrupamento } \\
\text { Valadares } \\
n=1286\end{array}$ & $\begin{array}{l}\text { Agrupamento } \\
\text { Vila d'Este } \\
n=484\end{array}$ \\
\hline $\begin{array}{c}42,86 \% \\
(n=186)\end{array}$ & $\begin{array}{l}32,89 \% \\
(n=50)\end{array}$ & $\begin{array}{c}38,82 \% \\
(n=269)\end{array}$ & $\begin{array}{l}33,46 \% \\
(n=85)\end{array}$ & $\begin{array}{l}54,41 \% \\
(n=37)\end{array}$ & $\begin{array}{c}34,04 \% \\
(n=436)\end{array}$ & $\begin{array}{c}33,98 \% \\
(n=437)\end{array}$ & $\begin{array}{c}37,81 \% \\
(n=183)\end{array}$ \\
\hline
\end{tabular}

\section{PROTECÇÃO DE PESSOAS E ANIMAIS}

Os autores declaram que os procedimentos seguidos estavam de acordo com os regulamentos estabelecidos pelos responsáveis da Comissão de Investigação Clínica e Ética e de acordo com a Declaração de Helsínquia da Associação Médica Mundial. O estudo foi elaborado de acordo com as International Ethical Guidelines for Biomedical Research Involving Humam Subjects, e conforme como proposto na metodologia do World Health Organization (WHO) European Childhood Obesity Surveillance Iniciative (COSI), que serviu de referência à construção metodológica do presente estudo.

\section{REFERÊNCIAS}

1. World Health Organization. Obesity and overweight. Fact Sheet $\mathrm{nr}$ 311. Geneva: WHO, Media Centre; 2015. [consultado 2015 mai 07]. Disponível em: http://www.who.int/mediacentre/factsheets/fs311/en/\#.

2. Abela S, Bagnasco A, Arpesella M, Vandoni M, Sasso L. Childhood obesity: an observational study. J Clin Nurs. 2014;23:2990-2.

3. Direção Geral da Saúde. Plataforma contra a obesidade. Lisboa; 2015. [consultado 2015 mai 07]. Disponível em: http://www. plataformacontraaobesidade.dgs.pt/.

4. de Onis M, Blössner M, Borghi E. Global prevalence and trends of overweight and obesity among preschool children. Am J Clin Nutr. 2010;92:1257-64.

5. World Health Organization. Obesity: preventing and managing the global epidemic. Report of a WHO consultation. World Health Organ Tech Rep Ser. 2000;894:1-253.

6. International Association for the Study of Obesity. Obesity: understanding and challenging the global epidemic. London: IASO; 2013.

7. Ng M, Fleming T, Robinson M, Thomson B, Graetz N, Margono C, et al. Global, regional, and national prevalence of overweight and obesity in children and adults during 1980-2013: a systematic analysis for the Global Burden of Disease Study 2013. Lancet. 2014;384:766-81.

8. World Health Organization. Food and Agriculture Organization of the United Nations. Diet, nutrition and the prevention of chronic diseases: report of a joint WHO/FAO expert consultation. Report No. 916. Geneva: WHO; 2003.

9. World Health Organization. Global status report on noncommunicable diseases 2010. Geneva: WHO; 2011.

10. Haddad L, Achadi E, Bendech MA, Ahuja A, Bhatia K, Bhutta Z, et al. The Global Nutrition Report 2014: Actions and Accountability to Accelerate the World's Progress on Nutrition. J Nutr. 2015;145:663-71.

11. Olds T, Maher C, Zumin S, Péneau S, Lioret S, Castetbon K, et al. Evidence that the prevalence of childhood overweight is plateauing: data from nine countries. Int J Pediatr Obes. 2011;6:342-60.

12. Krebs NF, Himes JH, Jacobson D, Nicklas TA, Guilday P, Styne D. Assessment of child and adolescent overweight and obesity. Pediatrics. 2007;120:S193-228.

13. Pulgaron ER. Childhood obesity: a review of increased risk for physical and psychological comorbidities. Clin Ther. 2013;35:A18-32.

14. Bingham DD, Varela-Silva MI, Ferrão MM, Augusta G, Mourao MI, Nogueira $\mathrm{H}$, et al. Socio-demographic and behavioral risk factors associated with the high prevalence of overweight and obesity in Portuguese children. Am J Hum Biol. 2013;25:733-42.

15. Padez C, Mourão I, Moreira P, Rosado V. Prevalence and risk factors for overweight and obesity in Portuguese children. Acta Paediatr.

\section{CONFIDENCIALIDADE DOS DADOS}

Os autores declaram ter seguido os protocolos do seu centro de trabalho acerca da publicação dos dados de doentes.

\section{CONFLITOS DE INTERESSE}

Os autores declaram a inexistência de conflitos de interesse relativamente ao presente artigo.

\section{FONTES DE FINANCIAMENTO}

Não existiram fontes externas de financiamento para a realização deste artigo.

2005;94:1550-7.

16. Hill JO, Peters JC. Environmental contributions to the obesity epidemic. Science. 1998;280:1371-74.

17. Beja A, Ferrinho $P$, Craveiro I. Evolução da prevenção e combate à obesidade de crianças e jovens em Portugal ao nível do planeamento estratégico. Rev Port Saúde Pública. 2014;32:10-17.

18. Wijnhoven T, van Raaij J, Sjöberg A, Eldin N, Yngve A, Kunešová M, et al. WHO European Childhood Obesity Surveillance Initiative: School Nutrition Environment and Body Mass Index in Primary Schools. Int J Environ Res Public Health. 2014;11:11261-85.

19. Lobstein T, Baur L, Uauy R. Obesity in children and young people: a crisis in public health. Obes Rev. 2004;5:4-85.

20. Langley-Evans SC, Moran VH. Childhood obesity: risk factors, prevention and management. Matern Child Nutr. 2014;10:453-5.

21. Commission of the European Communities. White paper on a Strategy for Europe on Nutrition, Overweight and Obesity related health issues. COM (2007) 279 final. Luxembourg: EC; 2007.

22. de Onis M, Lobstein T. Defining obesity risk status in the general childhood population: which cut-offs should we use? Int J Pediatr Obes. 2010;5:458-60.

23. Wijnhoven TM, van Raaij JM, Spinelli A, Rito AI, Hovengen R, Kunesova M, et al. WHO European Childhood Obesity Surveillance Initiative 2008 : weight, height and body mass index in 6-9-year-old children. Pediatr Obes. 2013;8:79-97.

24. European Commission. EU Action Plan on Childhood Obesity 2014 2020. Luxembourg: EC; 2014.

25. World Health Organization. European Food and Nutrition Action Plan 2015-2020. Copenhagen: WHO; 2014.

26. Assembleia da República. Lei n. $75 / 2013$ de 12 setembro: Regime jurídico das autarquias locais.

27. Borys JM, Le Bodo Y, Jebb SA, Seidell JC, Summerbell C, Richard D, et al. EPODE approach for childhood obesity prevention: methods, progress and international development. Obes Rev. 2012;13:299-315.

28. PORDATA. População residente segundo os Censos: total e por grandes grupos etários - Municípios 2011. [consultado 2015 mai 07]. Disponivel em: http://www.pordata.pt/.

29. Antunes A, Moreira P. Prevalência de excesso de peso e obesidade em crianças e adolescentes Portugueses. Acta Med Port. 2011;24:279-84.

30. Padez C, Fernandes T, Mourão I, Moreira P, Rosado V. Prevalence of overweight and obesity in 7-9-year-old Portuguese children: trends in body mass index from 1970-2002. Am J Hum Biol. 2004;16:670-8.

31. Pedrosa C, Correia F, Seabra D, Oliveira BM, Simões-Pereira C, Vazde-Almeida MD. Prevalence of overweight and obesity among 7-9-year- 
old children in Aveiro, Portugal: comparison between IOTF and CDC references. Public Health Nutr. 2011;14:14-9.

32. Rito A. Childhood Obesity Surveillance Initiative - COSI Portugal. Observações. Bol Epidem. 2012: 6.

33. Direção-Geral da Saúde. Programa Nacional para a Promoção da Alimentação Saudável: Orientações Programáticas. Lisboa: DGS; 2012.

34. Direção-Geral da Saúde. Plano Nacional de Saúde: Revisão e extensão a 2020. Lisboa: DGS; 2015.

35. Council for International Organizations of Medical Sciences. International Ethical Guidelines for Biomedical Research Involving Human Subjects Geneva: CIOMS; 2002

36. Wijnhoven T, Van Raaij J, Breda J. WHO European Childhood Obesity Surveillance Initiative: Implementation of round 1 (2007/2008) and round 2 (2009/2010). Copenhagen: WHO Europe; 2014.

37. World Health Organization. WHO child growth standards: training course on child growth assessment. Geneva: WHO; 2008

38. Lohman TG, Roche AE, Martorell R, editors. Anthropometric standardization reference manual. Illinois: Human Kinetic Books; 1988.

39. World Health Organization. AnthroPlus for personal computers Manual: Software for assessing growth of the world's children and adolescents. Geneva: WHO; 2009. [consultado 2015 Mai 07]. Disponível em: http:// www.who.int/growthref/tools/en/.

40. Rolland-Cachera MF. Childhood obesity: current definitions and recommendations for their use. Int J Pediatr Obes. 2011;6:325-31.

41. World Health Organization. Multicentre Growth Reference Study G, de Onis M. WHO Child Growth Standards based on length/height, weight and age. Acta Pædiatr. 2006;95:76-85.

42. Cole TJ, Lobstein T. Extended international (IOTF) body mass index cutoffs for thinness, overweight and obesity. Pediatr Obes. 2012;7:284-94.

43. Kuczmarski RJ, Ogden CL, Guo SS, Grummer-Strawn LM, Flegal KM Mei Z, et al. 2000 CDC Growth Charts for the United States: methods and development. Vital Health Stat 11. 2002:1-190.

44. Cole TJ, Bellizzi MC, Flegal KM, Dietz WH. Establishing a standard definition for child overweight and obesity worldwide: international survey. BMJ. 2000;320:1240-3.

45. de Onis M, Onyango AW, Borghi E, Siyam A, Nishida C, Siekmann J. Development of a WHO growth reference for school-aged children and adolescents. Bull World Health Organ. 2007;85:660-7
46. European Childhood Obesity Group. Endorsement of the new WHO Growth Standards for Infants and Young Children By the European Childhood Obesity Group. Brussels: ECOG; 2009.

47. International Pediatric Association. International Pediatric Association Endorsement: The New WHO Growth Standards for Infants and Young Children. Geneva: IPA; 2006

48. Cordinhã A, Paúl A, Fernandes L. Obesidade infantil e hipertensão arterial: a realidade de uma população pré-escolar. Acta Pediatr Port. 2009;40:145-9.

49. Lourenço $M$, Santos $C$, Carmo I. Estado nutricional e hábitos alimentares em crianças de idade pré-escolar. Rev Enf Ref. 2014;IV:7-14

50. Gonzalez-Casanova I, Sarmiento OL, Gazmararian JA, Cunningham SA, Martorell R, Pratt $M$, et al. Comparing three body mass index classification systems to assess overweight and obesity in children and adolescents. Rev Panam Salud Publica. 2013;33:349-55.

51. Ahrens W, Pigeot I, Pohlabeln H, De Henauw S, Lissner L, Molnar D, et al. Prevalence of overweight and obesity in European children below the age of 10. Int J Obes. 2014;38:S99-107.

52. Cattaneo A, Monasta L, Stamatakis E, Lioret S, Castetbon K, Frenken $\mathrm{F}$, et al. Overweight and obesity in infants and pre-school children in the European Union: a review of existing data. Obes Rev. 2010;11:389-98.

53. Nogueira H, Ferrão M, Gama A, Mourão I, Rosado Marques V, Padez C. Perceptions of neighborhood environments and childhood obesity: Evidence of harmful gender inequities among Portuguese children. Health Place. 2013;19:69-73.

54. OECD. Overweight and obesity among children. In Health at a Glance: Europe 2012. Paris: OECD; 2012

55. Rito A. Estado nutricional de crianças e oferta alimentar do pré-escolar do município de Coimbra, Portugal, 2001. Rio de Janeiro: Escola Nacional de Saúde Pública Sérgio Arouca da Fundação Oswaldo Cruz; 2004

56. Rito A, Wijnhoven TM, Rutter H, Carvalho MA, Paixão E, Ramos C, et al Prevalence of obesity among Portuguese children (6-8 years old) using three definition criteria: COSI Portugal, 2008. Pediatr Obes. 2012;7:41322.

57. World Health Organization. Vienna Declaration on Nutrition and Noncommunicable Diseases in the Context of Health 2020. Viena: WHO; 2013. 